\title{
Modeling the Interaction of Light Between Diffuse Surfaces
}

Cindy M. Goral, Kenneth E. Torrance, Donald P. Greenberg and Bennett Batta1le

Cornell University

Ithaca, New York 14853

\section{ABSTRACT}

A method is described which models the interaction of light between diffusely reflecting surfaces. Current light reflection models used in computer graphics do not account for the object-to-object reflection between diffuse surfaces, and thus incorrectly compute the global illumination effects. The new procedure, based on methods used in thermal engineering, includes the effects of diffuse light sources of finite area, as well as the "color-bleeding" effects which are caused by the diffuse reflections. A simple environment is used to illustrate these simulated effects and is presented with photographs of a physical model. The procedure is applicable to environments composed of 1 deal diffuse reflectors and can account for direct 11 lumination from a variety of light sources. The resultant surface intensities are independent of observer position, and thus environments can be preprocessed for dynamic sequences.

CR Categories and Subject Descriptors: I.3.7 [Computer Graphics]: Three-Dimensional Graphics and Realism; I.3.3 [Computer Graphics]: Picture/Image Generation

\section{General Terms: Algorithms}

Additional Key Words and Phrases: Diffuse reflections, light reflection models, radiosity, shading, form factors

Permission to copy without fee all or part of this material is granted provided that the copies are not made or distributed for direct commercial advantage, the ACM copyright notice and the title of the publication and its date appear, and notice is given that copying is by permission of the Association for Computing Machinery. To copy otherwise, or to republish, requires a fee and/or specific permission.

\section{Introduction}

Most real environments consist primarily of surfaces which reflect light diffusely. In such environments, direct illumination and the object-to-object reflections between diffuse surfaces may account for the overwhelming proportion of the total light energy in an environment. Current light reflection models used in computer graphics do not account for the interaction between diffuse surfaces, and thus incorrectly compute the global illumination effects.

In order to generate images which realistically simulate an actual scene, the physical behavior of visible light as it is propagated through an environment must be modeled. Since the intensity and distribution of light in a scene are governed by energy transport and conservation principles, these must be considered if one wishes to accurately simulate different light sources and materials in the same scene.

This paper describes a method which can be used to determine the intensity of light diffusely reflected within an environment. The method is based on energy principles and may be applied monochromatically or to finite wavelength intervals. The key assumption is that all surfaces are ideal diffuse (Lambertian) reflectors. The procedure is applicable to arbitrary environments composed of such surfaces, and it can account for direct illumination from a variety of light sources and all multiple reflections within the environment. A major advantage of the method is that the resultant surface intensities are independent of observer position. Thus, environmental intensity information can be preprocessed for dynamic sequences. Furthermore, since small specular areas may contribute little to the total light energy, such surface reflections can later be added to the diffuse reflection solutions with minimal error.

The initial realistic image synthesis approaches for raster displays were concerned primarily with the visible surface determination of polygonal environments. Early algorithms assumed diffuse (Lambertian) reflections to determine the color of the displayed polygons. In 1973, Phong [7] proposed a reflection model for the determination of the color of each pixel as a function of the direction of the surface normal. The Phong 
reflection model has been significant in the evolution of realistic image synthesis methods and is still widely in use. The formulation includes ambient and diffuse terms that provide surface color and shading, and a specular term that provides realistic highlights from direct 1 ight source reflections. Based upon the Torrance-Sparrow reflection model [11], Blinn [2] suggested improvements which recognized that the magnitude of the specular component is related to the intensity that reaches the surface from the mirror direction.

Cook and Torrance [3] proposed a reflection model that describes the behavior of light in terms of energy equilibrium and electromagnetic wave theory. Application of this model results in a very realistic appearance when rendering a wide variety of materials with varied surface finishes. Unfortunately, the model requires spatial integration of the global illumination information to provide the incident energy on a surface. None of the present methodologies for image synthesis are able to generate the information required for application of this model to situations other than an isolated object suspended in space.

In an attempt to solve the global 111 umination problem, the ray tracing methodology was introduced by whitted [13]. Ray tracing is used as a method of determining the global illumination information that is relevant to the image plane [6]. This method traces a ray from the eye through each pixel into the environment and generates new reflected and/or refracted rays at each surface a ray strikes. The reflection models employed to date in ray tracing approaches are empirical in nature and do not account for the required energy conservation conditions. Furthermore, the ray tracing methodology, which inherently provides only point-sampled information, is not sufficient for the application of energy equilibrium models to light behavior. Lastly, due to the "tree of rays" approach, only the intra-environment specular effects are considered.

Many existing reflection models require the addition of an ambient or background illumination term. The magnitude of this ambient term is usually specified arbitrarily. The procedure described in this paper correctly accounts for

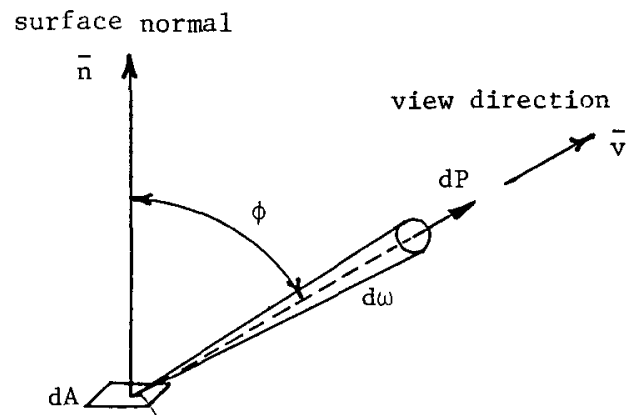

Figure 1. Geometry of Radiation Leaving a Surface. not only the "global ambient" term $[7,13,6]$, but also the object-to-object reflection between diffuse surfaces. In section 2, the theory and mathematical formulations are presented. Section 3 describes the program implementation. Resultant images are shown in section 4 and photographic results of a physical model appear in section 5 .

\section{Theory And Mathematical Formulation}

This section describes a method for determining the magnitude and color composition of light reflected within an environment. The major assumption is that all surfaces are ideal diffuse (i.e., Lambertian) reflectors. Illumination sources and surface reflective properties can be arbitrary within this constraint. The analysis, which is explained below, is similar to that used in thermal engineering for the calculation of radiative heat exchange in enclosures $[8,10,14]$.

The analytical procedure is built up by first introducing the concept of radiant intensity. Radiant energy in the form of visible light is presumed to emanate in all directions from a differential element of area, dA (Figure 1). The radiant intensity in a particular viewing direction is :

$$
i=d P /(\cos \phi d \omega)
$$

where,

$i$ = intensity of radiation in a particular viewing direction, expressed as the radiant energy leaving a surface per unit time per unit projected area (projected in the viewing direction) per unit solid angle (watts/meter**2 steradians)

$d P=$ the radiant energy leaving the surface in the direction $\phi$ within a solid angle $d \omega$ expressed per unit time and per unit surface area (unprojected) (watts/meter**2)

$\phi=$ polar angle measured from the surface normal to the viewing direction (degrees)

$d \omega=$ differential solid angle of the pencil of rays (steradians)

surface normal

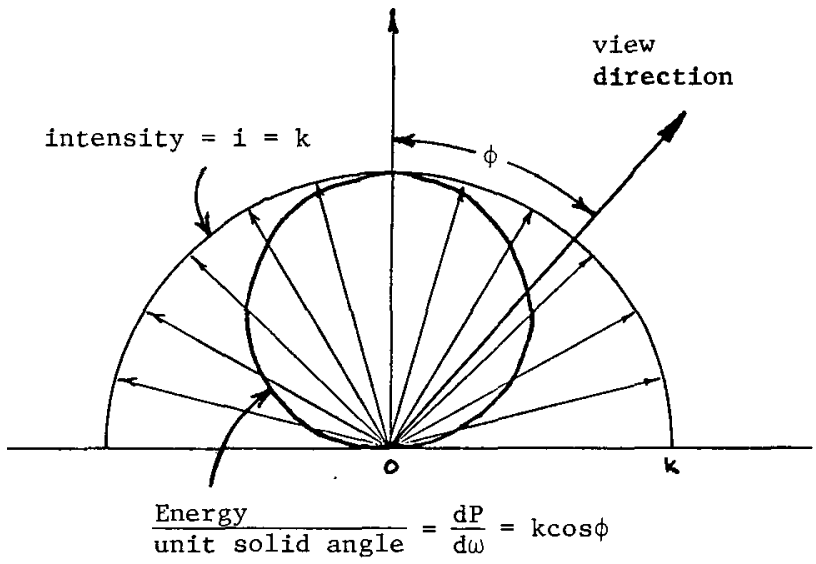

Figure 2. Ideal Diffuse Reflection from a Surface. 
The human eye senses Intensity; it perceives projected areas, and receives energy within a solid angle $d \omega$ defined by the pupil size. Intensity is thus an appropriate quantity for use in the construction of computer-generated images.

Next consider the properties of ideal diffuse or Lambertian reflection. For ideal diffuse reflection, the distribution of the reflected light energy is expressed by $d P / d \omega=k \cos \phi$, where $k$ is a constant. Since intensity is a function of the projected area, and the projected area varies with $\cos \phi$, the corresponding intensity $i$ of the reflected light is:

$$
i=\frac{\mathrm{dP} / \mathrm{d} \omega}{\cos \phi}=\frac{\mathrm{k} \cos \phi}{\cos \phi}=\mathrm{k}
$$

Thus, the intensity of the diffusely reflected light is constant and of uniform visual intensity from all viewing directions. Angular distributions of intensity and $\mathrm{dP} / \mathrm{d} \omega$ for an ideal diffuse reflector are shown in Figure 2 .

The total energy leaving the surface is found by integrating (1) over the hemisphere (solid angle $2 \pi$ ) above the surface:

$$
P=\int_{2 \pi} d P=\int_{2 \pi} 1 \cos \phi d \omega
$$

$P=$ total energy leaving the given surface and passing into the hemispherical space above the surface per unit time and area (watts/meter**2)

For an Ideal diffuse (Lambertian) surface, the total energy and intensity are related by:

$$
P=1 \int_{2 \pi} \cos \phi d \omega=i \pi
$$

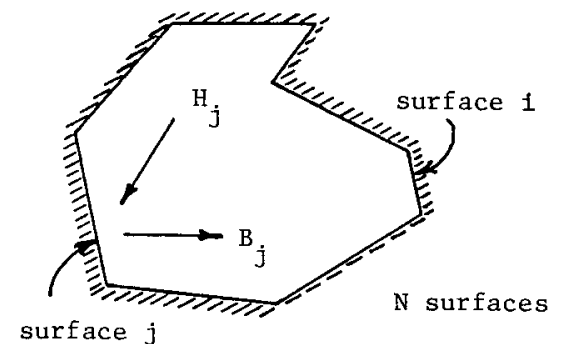

Figure 3. Enclosure Consisting of $N$ Surfaces. $H(j)$ and $E(j)$ Denote Incident and Emergent Fluxes for the $j$ th Surface.
Now consider the general problem of diffuse light reflection within an environment. Two concepts necessary for modeling the reflection of light are enclosures and form factors. A calculation of the light energy at any surface must include all of the radiation arriving at that surface from all directions in space. To account for the arriving radiation, a hypothetical enclosure is constructed. The enclosure is a set of surfaces that completely define the illuminating environment. The illumination and reflection properties of each surface of the enclosure must be specified. The walls of the enclosure consist of light sources and reflecting walls, and one or more of the surfaces of the enclosure may be fictitious (e.g., an open window). An $\mathrm{N}$-surface enclosure is sketched in Figure 3. The light arriving at a surface $j$, denoted by $H(j), \quad$ is found by summing the contributions from the other $\mathrm{N}-1$ surfaces, and from surface $j$ if it sees itself. The light emerging from the surface $j$ is denoted by $B(j)$.

All surfaces of the enclosure are assumed to be ideal diffuse reflectors, ideal diffuse light emitters, or a combination of the two. Each surface is assumed to be of uniform composition, with uniform illumination, reflection, and emission intensities over the surface. This assumption can generally be satisfied by subdividing the original surfaces of the enclosure. If diffuse light sources are used, such sources are treated as surfaces of the enclosure with specified illuminating intensities. If an arbitrary directional light source is used, the surfaces illuminated by the source are identified. The light di rectly reflected by these surfaces can be treated as diffuse light sources. For example, a spotlight which provides illumination over a finite area can be replaced by an equivalent diffuse illuminating panel. Isolux contours [12] can be computed for the panel, and each constant intensity region can be modeled as a separate diffuse light source. As a consequence, all reflected and illuminating light in the enclosure is diffuse, and can be combined for purposes of analysis.

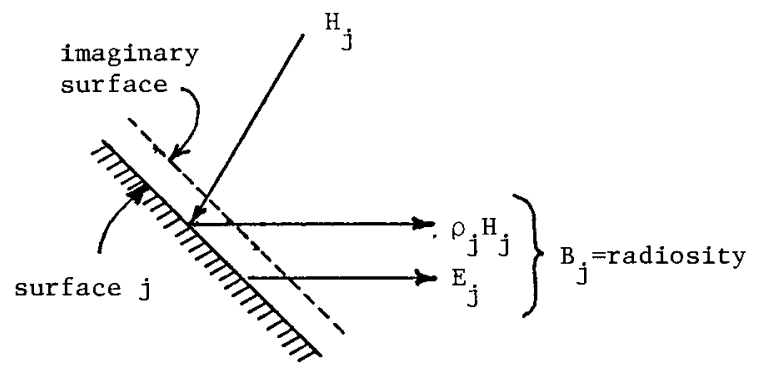

Figure 4. Details at a Particular Surface $j$. 
The second concept to be introduced is the form factor, F. It is defined as the fraction of the radiant light energy leaving one particular surface which strikes a second surface. The radiant light may have an arbitrary angular distribution of intensity. However, the most useful form factors are those for ideal diffuse emission or reflection. In this case, the form factor is purely geometrical in nature and is dependent only on the shape, size, position and orientation of the participating surfaces.

With the foregoing introduction, it is now possible to formulate expressions for the intensity of all the surfaces in an enclosure. Consider the surface $j$ in Figure 4. An imaginary surface is stretched above the actual surface, as shown by the dashed line. The radiosity $B(j)$ is the hemispherical integral of the energy leaving the surface. To an observer, the surface $j$ appears to be emitting a flux, $B(j)$, from the imaginary surface. This flux consists of two parts given by:

$$
B_{j}=E_{j}+\rho_{j} H_{j}
$$

where,

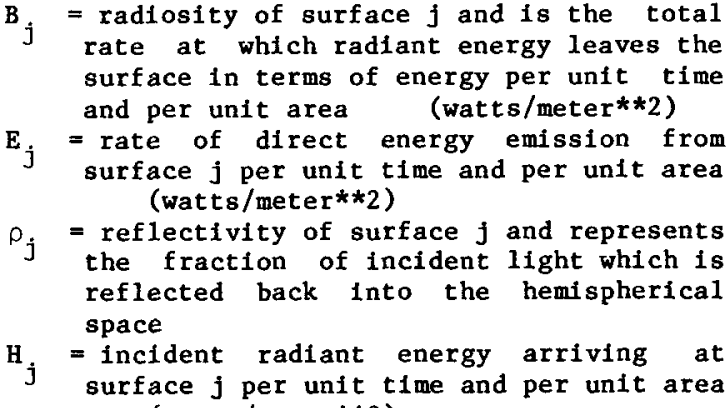
(watts/meter**2)

However, the observer sees a total flux $B(j)$, and is unable to distinguish between the two components on the right side of equation (4) because they both have the same directional distribution in space (i.e., diffuse). Thus, there is no need to treat the emitted and reflected radiation separately. The analysis is simplified by considering only one quantity, $B(j)$. There is a radiosity $B(j)$ for each surface in the enclosure.

Consider next the incident $f \operatorname{lux} H(j)$ on surface $j$ in Figure 3. This is the sum of fluxes from all surfaces in the enclosure that "see" $j$. The fraction of the flux leaving surface $i, B(i)$, and reaching surface $j$ is specified by the form factor, $F(i j)$. Since all surfaces contribute to the irradiation onto $j$, the incident $f$ lux $i s$ found by summing the contributions from all surfaces:

$$
H_{j}=\sum_{i=1}^{N} B_{i} F_{i j}
$$

\footnotetext{
$B$ = radiosity of surface 1 (watts/meter $\star * 2$ )

$\mathrm{F}=$ form factor and represents the fraction of radiant energy leaving surface 1 and impinging on surface $j$
}

The summation Includes surface $j$ because surface $j$ might see Itself (1.e., $F(i i)$ need not be zero if, for example, the surface is concave). Combining equations (4) and (5) results in:

$$
B_{j}=E_{j}+\rho_{j} \sum_{i=1}^{N} B_{1} F_{1 j} \text { for } j=1, N
$$

Such an equation exists for every surface in the enclosure. Since the procedure is based on computing radiosities, within this paper it is referred to as the "radiosity method". The radiosity formulation accounts for all light leaving and incident upon a surface. The incident light is simply expressed in terms of the incident radiosity; no further ray tracing is needed to account for all interchanges (including multiple reflections) in an enclosure.

In general, equation (6) yields a set of $N$ linear equations with $N$ unknown $B(j)$ values, containing parameters $E(j), \rho(j)$, and $F(1 j)$ which must be known or calculated for each surface. The emission terms, $E(j)$, represent the 1 llumination sources for the system. If all the $E(j)$ 's are zero, there is no illumination and $a 11$ the $B(j)$ 's are zero. The $E(j)$ 's are nonzero only at surfaces that provide illumination to the enclosure. Such surfaces could represent a diffuse lllumination panel, or the first reflection of a directional light source from a diffuse surface. The $E(j)$ 's are thus determined by the conditions of illumination, and represent the external source terms.

A system of equations of the form of equation (6) may be applied monochromatically, for any finite bandwidth of radiation, or over the entire visible spectrum (provided that the wavelength-dependent quantities $E(j)$ and $\rho(j)$ are appropriately-defined average values). This result follows because none of the visible light in the enclosure, defined by the $B(j)$ 's, is absorbed by the walls and reradiated back into the enclosure. As a result, the $E(j)$ 's and $B(j)$ 's are effectively uncoupled, and the $E(j)$ 's may be specified independently.

For synthetic Image generation, radiant intensity rather than radiant energy is computed since the eye senses intensity. Since all the radiant energy terms in equation (6) are diffuse in character, they may be converted to radiant intensities by simply dividing by $\pi$ (see equation (3a)). Dividing by $\pi$, and denoting the radiant intensities corresponding to $B(j)$ and $E(j)$ by $b(j)$ and $e(j)$, respectively, yields:

$$
b_{j}=e_{j}+p_{j} \sum_{i=1}^{N} b_{j} F_{i j} \text { for } j=1, N
$$

\subsection{Form Factors}

In order to determine the form factors for radiative exchange between two finite surfaces with areas $A(i)$ and $A(j)$, first consider the form factors for exchange between two infinitesimal surfaces with differential areas $d A(i)$ and $d A(j)$ 
(Figure 5). Consistent with the assumptions made earlier, all the reflected and emitted light leaving a surface is assumed to be diffusely distributed. As seen from $\mathrm{dA}(1)$, the solid angle subtended by $\mathrm{dA}(\mathrm{j})$ is:

$$
\mathrm{d} \omega=\frac{\cos \phi_{j} \mathrm{dA} j}{\mathrm{r}^{2}}
$$

Using equations ( 1 ) and ( $3 a$ ), the radiant energy leaving $d A(i)$ directly incident on $d A(j)$ is :

$$
\mathrm{dP}_{1} \mathrm{dA}_{1}=i_{1} \cos \phi_{1} \mathrm{~d} \omega \mathrm{dA} A_{1}=\frac{\mathrm{P}_{1} \cos \phi_{1} \cos \phi_{j} \mathrm{dA}_{i} \mathrm{dA}_{j}}{\pi \mathrm{r}^{2}}
$$

The total energy leaving surface $d A(i)$ into the hemisphere is $P(i) d A(i)$. Noting that the form factor represents the fraction of the total energy emanating from dA(i) which is directly incident on surface $d A(j)$, one obtains:

$$
\begin{aligned}
F_{d_{1}-d A_{j}} & =\frac{P_{i} \cos \phi_{i} \cos \phi_{j} d_{i} d_{j} / \pi r^{2}}{P_{i} d_{i}} \\
& =\frac{\cos \phi_{1} \cos \phi_{j} d A_{j}}{\pi r^{2}}
\end{aligned}
$$

It is evident that the value of the form factor is proportional to the infinitesimal area $\mathrm{dA}(j)$. To compute the fraction of radiation leaving $\mathrm{dA}(1)$ and reaching the finite area $A(j)$,

$$
F_{d A_{i}-A_{j}}=\int_{A_{j}} \frac{\cos \phi_{i} \cos \phi_{j} d_{j}}{\pi r^{2}}
$$

The form factor between the finite surfaces, $A(i)$ and $A(j)$, is defined as the area average of equation (11):

$$
F_{A_{i}-A_{j}}=F_{i j}=\frac{1}{A_{1}} \int_{A_{1} A_{j}} \int_{\frac{\cos \phi_{i} \cos \phi_{j} d A_{i} d A_{j}}{\pi r^{2}}}
$$

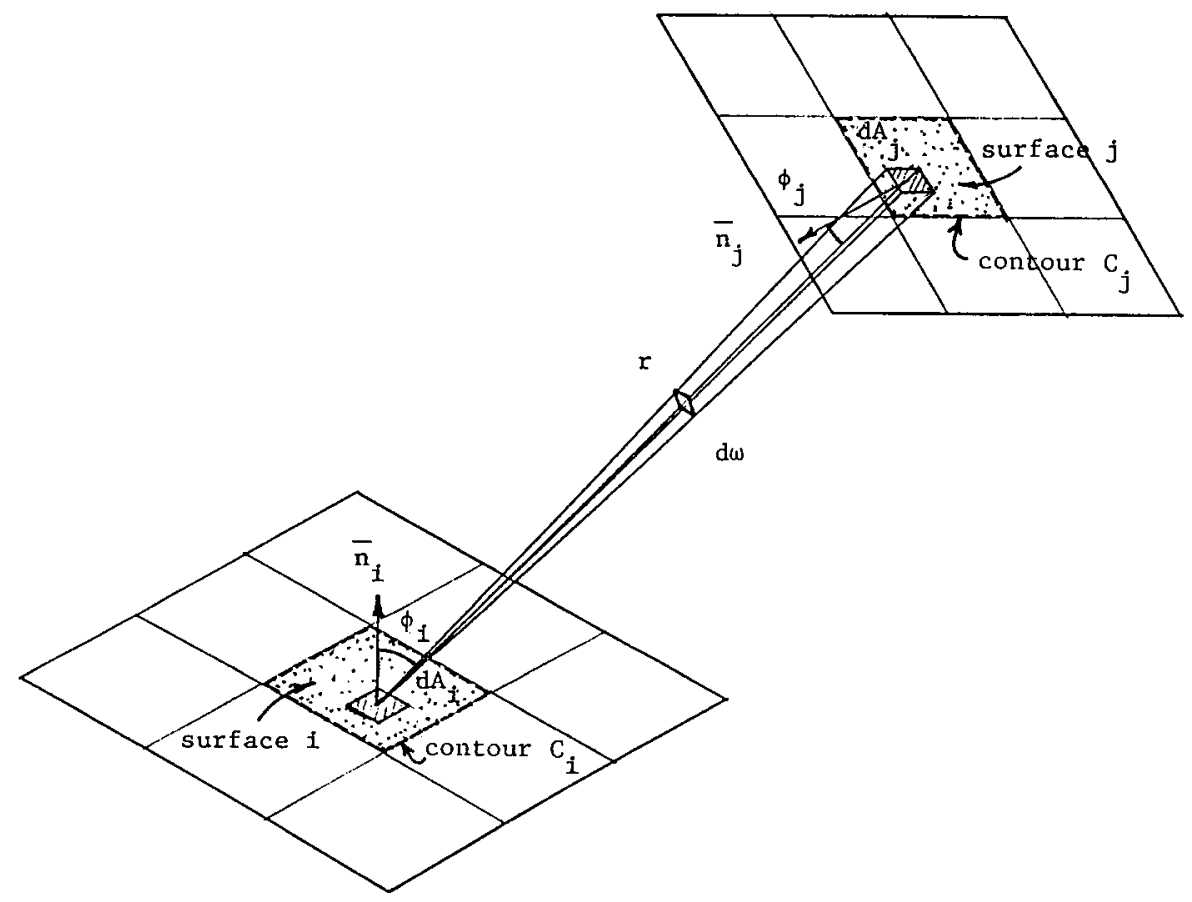

$\mathrm{dA}(j)=$ elemental area on surface $j$

$A(j)=$ area of surface $j$

$C(j)=$ contour of surface $j$

$C(i)=$ contour of surface $i$

$r=$ distance between $\mathrm{dA}(i)$ and $\mathrm{dA}(\mathrm{j})$

$\mathrm{d} \omega=$ solid angle subtended by $\mathrm{dA}(j)$ as seen from dA(i)

$\phi(i)=$ angle between surface normal of $i, \bar{n}(i)$, and the line

$\phi(j) \quad=$ angle between surface normal of $j, \bar{n}(j)$, and the line $r$

Figure 5. Geometry for Form Factor Derivation 
In equation (12), the form factor is expressed as a double area integral. There are more efficient methods of computing form factors. One method is the contour integral representation which is obtained by transforming the area integrals into contour integrals using Stokes' theorem $[9,10]$.

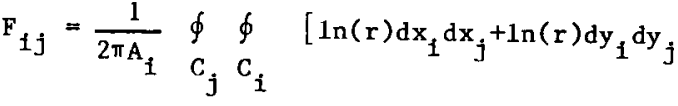

$$
\begin{aligned}
& \left.+\ln (r) d z_{i} d z_{j}\right]
\end{aligned}
$$

Equation (13) above was used in our implementation.

From the formulation of the form factors, some simple identities which also serve as checks or shortcuts for calculations can be derived:

1. A reciprocity relationship can be derived from (12) for radiosity distributions which are diffuse and uniform over each surface:

$$
A_{i} F_{1 j}=A_{j} F_{j i}
$$

A knowledge of $F(i j), A(i)$ and $A(j)$ thus allows $F(j i)$ to be determined.

2. In order to achieve conservation of energy in a closed environment of $N$ surfaces, all of the energy leaving a surface must be accounted for. Thus, the form factors for each surface must sum to unity:

$$
\sum_{j=1}^{N} F_{i j}=1 \quad \text { for } 1=1, N
$$

3. For a plane or convex surface (one that does not see itself),

$$
\mathrm{F}_{11}=0
$$

For an enclosure with $N$ surfaces, the matrix containing the form factors $F(i j)$ has $N^{* * 2}$ elements, but many of the elements can often be found by using equations (14), (15), and (16).

\section{Program Implementation}

A program has been implemented which demonstrates the radiosity approach described above. The program reads an environment description, subdivides the polygons of the environment into subpolygon elements, computes the form factors between the elements, and forms and solves the matrix version of equation (7) to obtain element intensities. It then performs intensity smoothing between elements and displays the resultant image.
The program is limited to convex, polygonal surfaces. The present implementation is restricted to quadrilaterals and did not account for hidden surfaces.

The first module reads a description of a polygonal environment. This information consists of vertex coordinates, and the reflectivity and emitted energy terms for each color band for each polygon (equation 7). Three constant spectral energy bands, which approximated the red, green, and blue primary colors of the display device were used.

The second module subdivides the polygonal surfaces of the environment into subpolygons called "elements". This procedure is similar to mesh generation in finite element analysis [5]. A subdivision routine has been implemented where the number of elements for each polygonal surface is an input parameter to the program.

Form factors are then computed between all pairs of elements. The smaller the elements, the more accurate the results, but the longer the computation time. For the evaluation of form factors, the contour integration method (equation 13) was used. To discretize the contour integral, each edge of each element is divided into an equal number of segments. The approximate contour integral between elements $i$ and $j$ can then be expressed as a summation by the following pseudo-program:

/* FF is used to accumulate the form factor value */ $\mathbf{F F}:=0$

for each segment of the perimeter of element $i$ : \{

for each segment of the perimeter of element $j$

evaluate the distance between the segments; take the natural $\log$ of the distance; evaluate the lengths of the segments along each axis $\left(d x_{i}, d x_{j}, d y_{i}, d y_{j}, d z_{i}, d z_{j}\right)$;

multiply the natural $\log$ by $\left(d x_{i} d x_{j}+d y_{i} d y_{j}+d z_{i} d z_{j}\right)$;

add the result to $\mathrm{FF}$; ]

divide FF by $2 \pi$ times the area of element 1 ; /* FF is now an approximation to the form factor */ $/ *$ from element $i$ to element $j$

The actual program makes two extensions to the above algorithm. First, the approximate integral over each segment is evaluated with a quadratic (three-point) open formula. The double contour integration leads to a nine-point two dimensional quadrature formula $[1$, p. 892]. Second, when segments $11 \mathrm{e}$ on the same line, the integral is evaluated analytically both to improve the accuracy of the integration of $\ln (r)$ and to avoid the singularity when $r$ goes to zero. 
After the form factors have been computed, the matrix which determines intensity is formed and solved. To solve the radiosity equations of (7) for $N$ surfaces, the matrix is:

$\left[\begin{array}{cccc}1-\rho_{1} F_{1,1} & -\rho_{1} F_{1,2} & \cdots & -\rho_{1} F_{1, N} \\ -\rho_{2} F_{2,1} & 1-\rho_{2} F_{2,2} & \cdots & -\rho_{2} F_{2, N} \\ \vdots & & & \\ -\rho_{N} F_{N, 1} & -\rho_{N} F_{N, 2} & \cdots & 1-\rho_{N} F_{N, N}\end{array}\right]\left[\begin{array}{l}b_{1} \\ b_{2} \\ \vdots \\ b_{N}\end{array}\right]=\left[\begin{array}{l}e_{1} \\ e_{2} \\ \cdot \\ e_{N}\end{array}\right]$

The unknowns are the intensities, $b(1)$ 's. The matrix system must be set up each time the reflectivities ( $\rho^{\prime} s$ ) change. Since the form factors are a function of geometry only, they remain the same for each wavelength and need only be computed once. Note that if only the emitted intensities are changed (i.e., e's), the matrix remains the same.

Once the matrix has been established, any standard matrix solver can be used to derive the resulting radiosities. A Gaussian elimination scheme with partial pivoting was used.

Because the polygons are subdivided into elements of constant color larger than pixel resolution, a smoothing routine can enhance the quality of the image. Initially, a linear smoothing of the elements across the screen projection of each original polygon was implemented [4].

The final step is the display of the image. The images are displayed on a 3-channel, 27-bit, $512 \times 480$ resolution Grinnell frame buffer. The program was written in $C$ on a VAX 11/780 under VMS.

\section{Results}

A simulated environment consisting of the interior of a cube was used to illustrate the radiosity method. One wall was modeled as a diffuse light source and the other five were modeled as diffuse reflectors. One wall was red, one wall was blue, and the top, bottom, and wall facing the light source were gray. The reflectivity $(\rho)$ and intensity of emission(e) of each surface were specified in three RGB wavelength bands as shown in Figure 9a.

The six surfaces of the cube were each divided into $n$ elements and the associated form factors were computed. Using the nomenclature of the previous section, the walls were treated as polygons, and the polygons were subdivided into equal area square elements. The pictures in Figure 6 show the effect of subdividing the walls (polygons) into 1, 4, 9, 16,25 , and 49 elements. The figures illustrate that the more subdivisions, the more realistic the simulation. The graphs of the red, green, and blue intensities on a given scanline show the correct interaction and "color bleeding" of the red and blue walls on the gray walls. This effect can not be simulated using previous reflection models. It is instructive to compare the radiosity method to conventional diffuse shading models. Current light reflection models compute the intensities of diffuse surfaces usually by assuming point light sources located at an infinite distance. This assumption results in a constant intensity per polygon. If the light source is positioned at a finite distance, the computed intensity varies across the polygonal surface. None of the current models consider the effect caused by a light source of finite area, i.e., an "area source" as contrasted to a point source [12].

Figure 7 depicts a series of pictures, with each wall subdivided into twenty-five elements. The pictures show a progression of 1mages computed by another program. In this progression, the number of multiple reflections is successively increased. The first picture depicts an image in which each element is illuminated only by the diffuse "area light source" on the front wall. There are no red or blue contributions from the side walls onto the other surfaces. This is equivalent to using Lambert's law for an area light source and not allowing object-to-object reflections. Figures $7 b, c, d, e$ depict the results of adding one, two, four, and eight intermediate reflections, respectively. Figure $7 e$ is visually identical to the result obtained using the radiosity approach (Figure $7 \mathrm{f}$ ), where all possible illumination paths have been included. The RGB scanline intensity plots show not only the interaction of the walls and the "color bleeding", but also the fact that the overall picture brightness increases with the number of internal reflections.

Figures $8 \mathrm{a}$ and $8 \mathrm{~b}$ use the same constant element intensities as in Figures $6 \mathrm{c}$ and $6 \mathrm{f}$, respectively, but linear interpolation [4] was used to smooth the spatial variation of intensities prior to display generation. Linear interpolation, as contrasted to further element subdivision, is a reasonable choice for improving the representation of the true diffuse reflection behavior.

\section{Comparison With Physical Model}

To qualitatively verify the theoretical results by comparison with a real environment, a physical model of a simple environment was constructed and photographed. Fiber board panels, painted with flat latex paints to minimize specular reflections, were used to construct a test cube (Figure 9b). This cube consisted of one red, one blue, and three white panels. One side was left open for viewing and photographic purposes.

In order to verify the reflective properties of the physical model, separate tests were conducted in which individual wall panels were 111 uminated with a paralle1 beam of incident 1ight. The intensity of the reflected light was measured as a function of reflection angle and angle of incidence. For near normal illumination $(\phi<60)$, essentially ideal diffuse behavior was observed. Equipment was not available for measuring the RGB reflectivities of the paints for comparison with the values used for the simulation (see Figure 9a). This precludes 


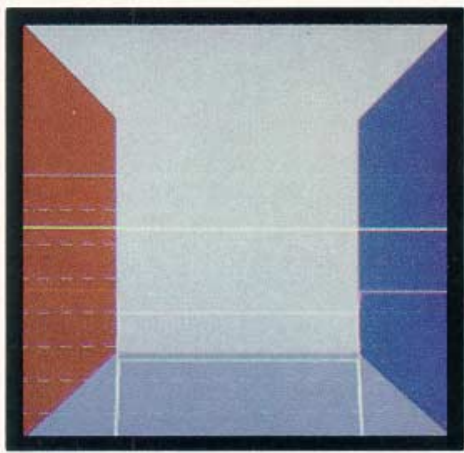

(a) 1 patch per side

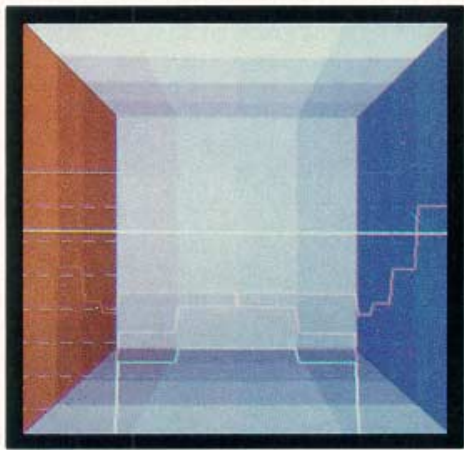

(d) 16 patches per side

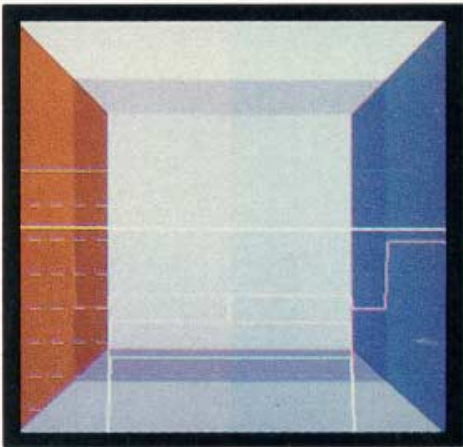

(b) 4 patches per side

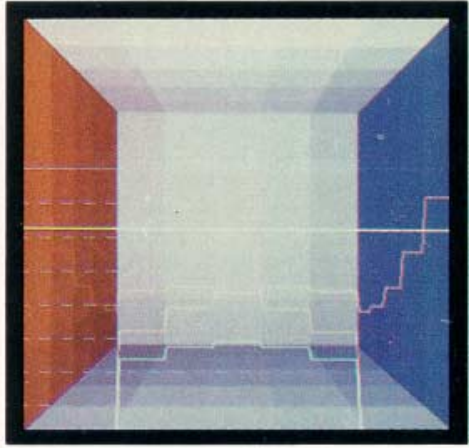

(e) 25 patches per side

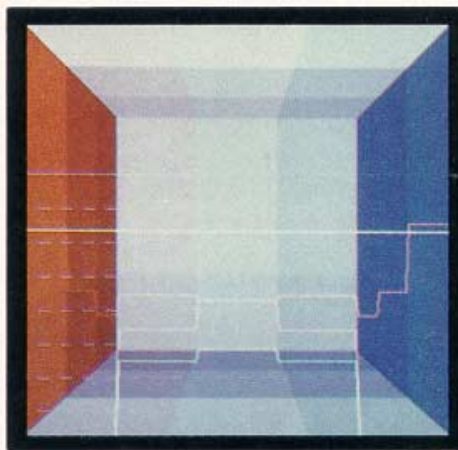

(c) 9 patches per side

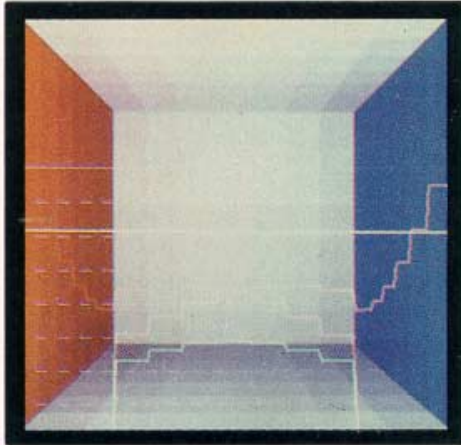

(f) 49 patches per side

Figure 6. Simulated Cube with Varying Wall Subdivisions and Constant Element Intensity. BGB Intensity Distributions at Mid-height Scanline are Shown.

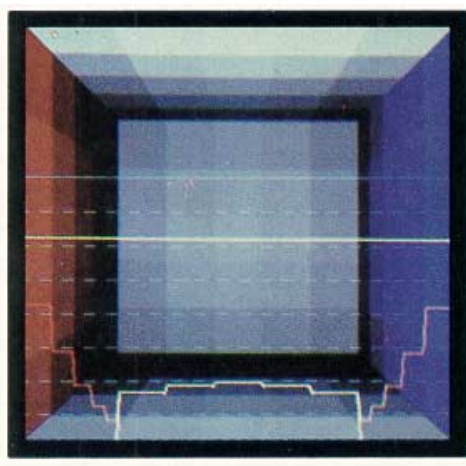

(a) 0 internal reflections

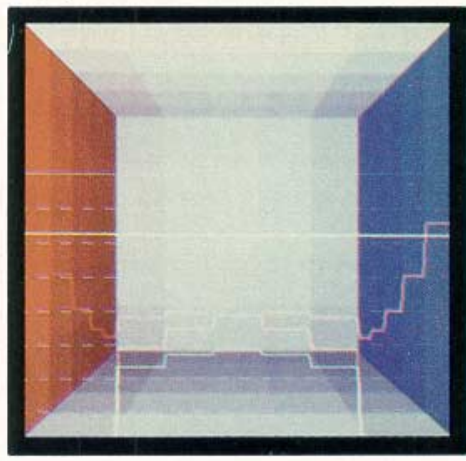

(d) 4 internal reflections

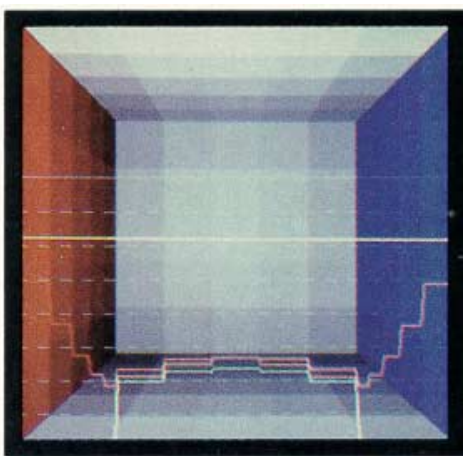

(b) 1 internal reflection

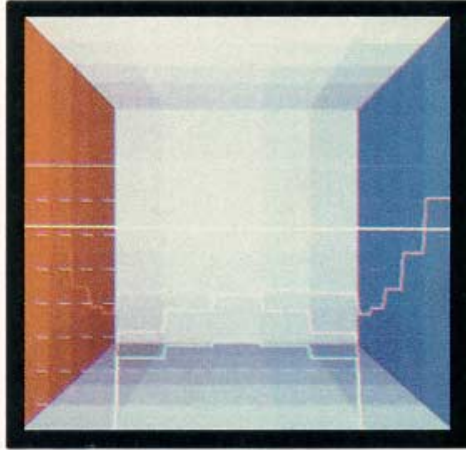

(e) 8 internal reflections

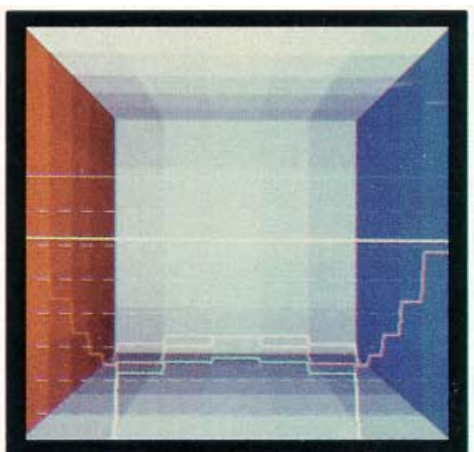

(c) 2 internal reflections

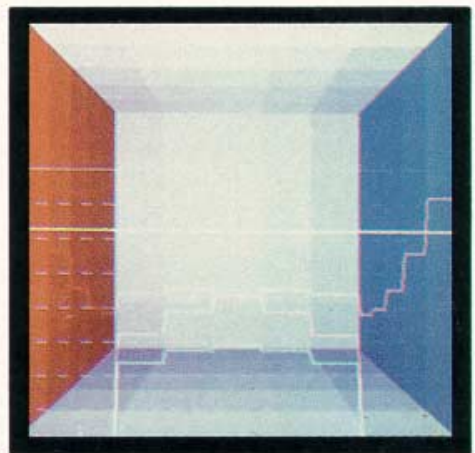

(f) Using Radiosity

Figure 7. Simulated Cube Showing the Effect of Increasing the Number of Reflections, 25 patches per side. 

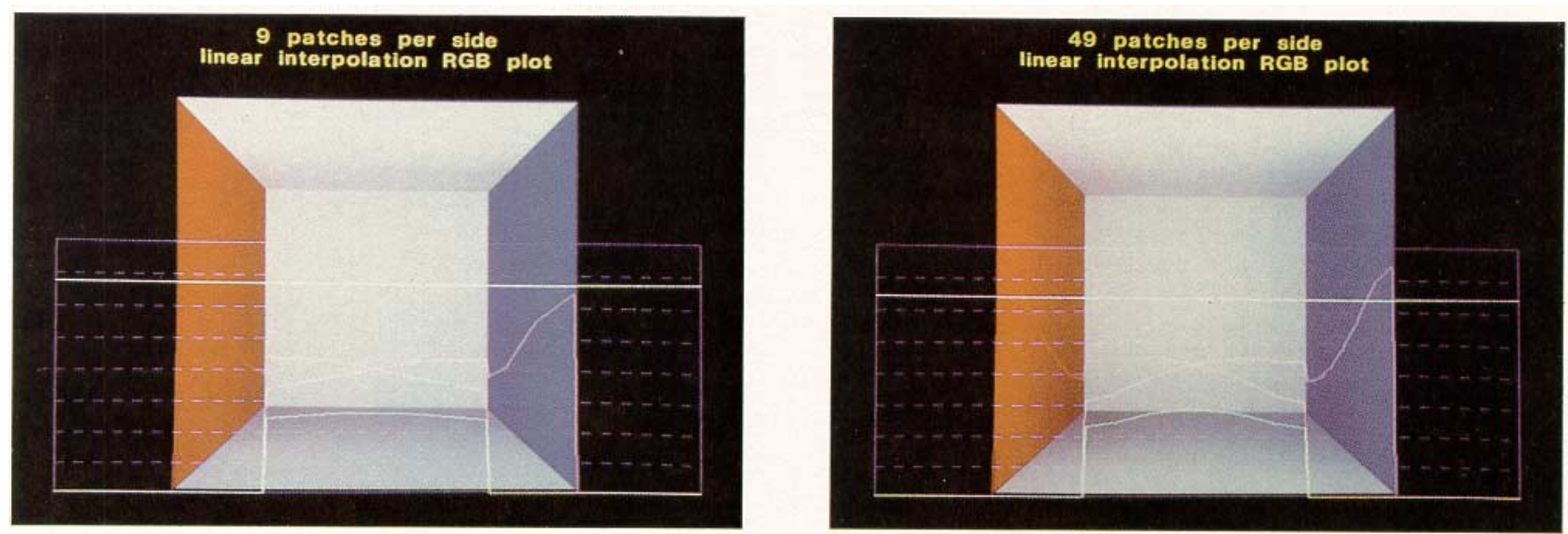

(a)

Figure 8. Simulated Cube with Two Wall Subdivisions and Linear Interpolation Over each Element (Patch).
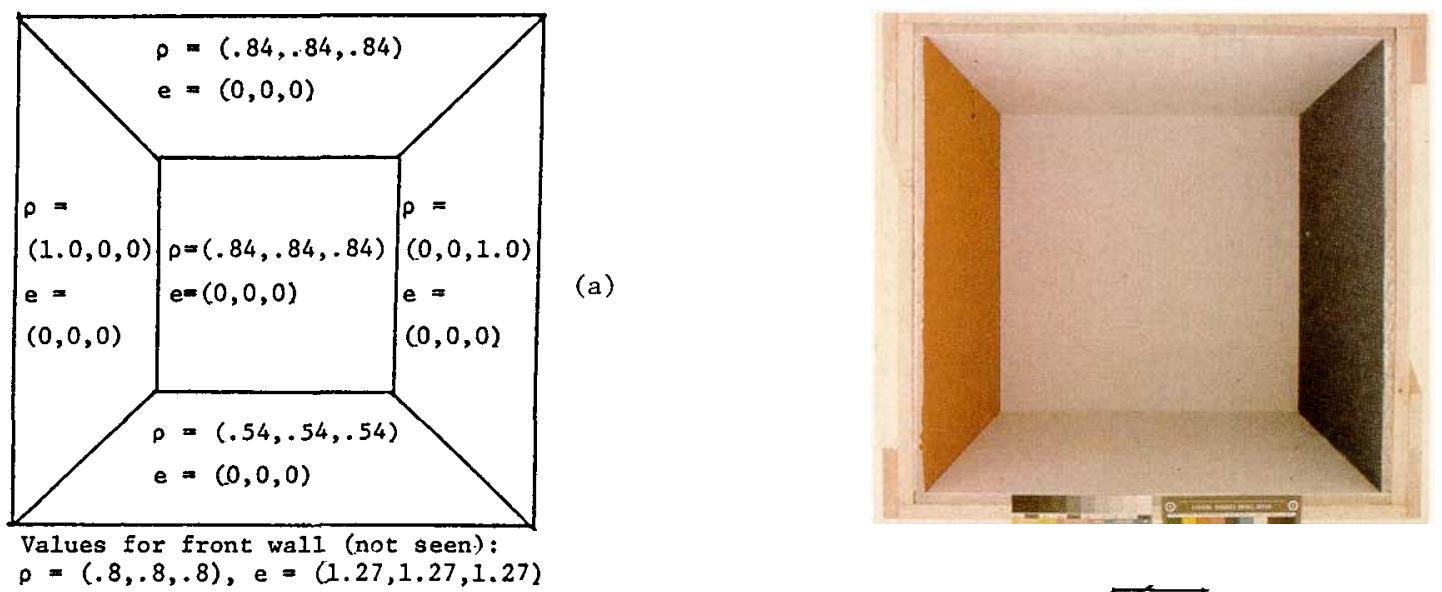

(b)

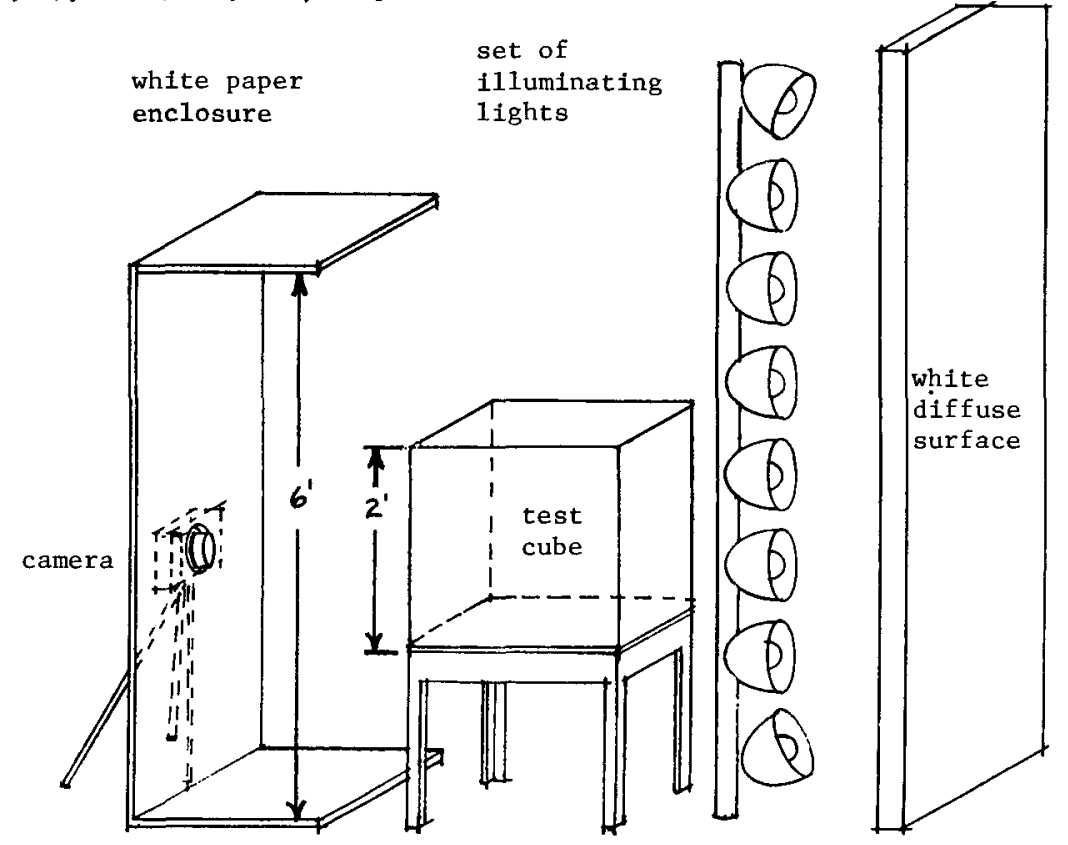

(c)

Figure 9. Diagram of Experimental Test. Reflectivity and Emissivity Values of Simulated Mode1 are Shown in (a). Photograph of Real Mode1 (b). Schematic of Environment (c). 
quantitative comparison between simulated and real models.

For the physical model, the open face of the test cube was illuminated with diffuse white 1ight. A second larger enclosure with white inside walls and an open side faced the cube. The diffuse white light was obtained by flluminating this second enclosure with white lights (Fig. 9c). Through a small hole cut in the second enclosure, photographs of the cube's interior were taken. This allowed the pictures to be taken without interfering with the diffuse lighting requirements for the experiment. The illuminating wall was isotropic and uniform to approximately ten percent.

A photograph of the real model is shown in Figure $9 \mathrm{~b}$. The most significant observation is the color-bleeding on the top, bottom, and back walls. This color-bleeding is apparent in the simulated images using the radiosity approach (Figures 6 and 8), but not in Figure 7a, which displays the effect of neglecting object-to-object multiple reflections.

\section{Conclusions}

A method has been described which models the correct interaction and object-to-object reflections between diffusely reflecting surfaces. Current light reflection models used in computer graphics do not account for this interaction, and thus incorrectly compute the global illumination effects. The procedure explicitly contains the effects of diffuse light sources of finite area, as well as the "color-bleeding" effects which are caused by the diffuse reflections.

Although computationally expensive, the procedure has a major advantage in that the results are independent of the observer position. Once the intensities have been computed for a static environment, the scene can be displayed from any position without recomputing intensity values. Thus, environmental intensities can be preprocessed for dynamic sequences. Furthermore, since small specularly reflecting objects may contribute little to the total light energy, the effects of such specular reflections can be superimposed on the diffuse solutions with minimal error.

Future work should include creating a smarter subdivision algorithm to obtain finer meshes in regions of high intensity gradients and considering occluded surfaces and non-polygonal objects.

\section{Acknowledgements}

This research was performed at the Program of Computer Graphics at Cornell University and supported by the National Science Foundation under grant number MCS8203979. Thanks go to Michael Cohen, Kevin Koestner, and Tim McCorry for their assistance in the model building, to Dottie Harrelson for typing, to Phil Brock for drawings, and to Emil Ghinger for photography. Thanks also go to an anonymous reviewer for providing many helpful comments.
8 References

[1] Abramowitz, Milton and Stegun, Irene (Ed.). Handbook of Mathematical Functions with Formulas, Graphs, and Mathematical Tables. US Dept of Commerce Nationa1 Bureau of Standards, Applied Mathematics Series 55, June 1964.

[2] Blinn, James F. Models of light reflection for computer synthesized pictures. ACM Computer Graphics (Siggraph Proc 177) 11, 2, (Summer 1977), 192-198.

[3] Cook, Robert L. and Torrance, Kenneth E. A reflectance model for computer graphics. ACM Computer Graphics (Siggraph Proc '81) 15, 3, (August 1982), 307-316.

[4] Gouraud, Henri. Computer display of curved surfaces. $\mathrm{PhD}$ dissertation, University of Utah, Salt Lake City, 1971.

[5] Haber, Robert, Shepard, Mark, Abe1, John, Gallagher, Richard and Greenberg, Donald. A generalized graphic preprocessor for two-dimensional finite element analysis. ACM Computer Graphics (Siggraph Proc '78) 12, 3, (August 1978), 323-329.

[6] Ha11, Roy and Greenberg, Donald P. A testbed for realistic image synthesis. IEEE Computer Graphics and Applications 3, 8, (November 1983), $10-20$.

[7] Phong, Bui Tuong. Illumination for computer-generated images. PhD dissertation, University of Utah, Salt Lake City, 1973.

[8] Siegel, Robert and Howe11, John R. Thermal Radiation Heat Transfer. Hemisphere Publishing Corporation, Washington, 1981.

[9] Sparrow, E.M. A new and simpler formulation for radiative angle factors. Transactions of the ASME, Journa1 of Heat Transfer 85,2 , (1963), $81-88$.

[10] Sparrow, E.M. and Cess, R.D. Radiation Heat Transfer. Hemisphere Publishing Corporation, Washington, 1978.

[11] Torrance, Kenneth E. and Sparrow, Ephraim M. Theory for of $f$-specular reflection from roughened surfaces. Journal Optical Society of America 57, 9, (September 1967), 1105-1114.

[12] Verbeck, Channing P. and Greenberg, Donald P. A comprehensive light source description for computer graphics. submitted for publication, 1984.

[13] Whitted, Turner. An improved illumination model for shaded display. Communications of the ACM 6, 23, (June 1980), 343-349.

[14] Wiebelt, John A. Engineering Radiation Heat Transfer. Holt, Rinehart and Winston, Inc., New York, 1966. 